\title{
PLASMOBLASTIC LYMPHOMA WITH MAMMARY MANIFESTATION: A CASE REPORT
}

Rodrigo Quevedo de Lima'1, Eimi Nascimento Pacheco', Luiz Henrique Santana de Araujo', Cassio Fernando Paganini', Katsuki Arima Tiscoski'

'Santa Casa de Misericórdia de Porto Alegre - Porto Alegre (RS), Brazil.

Introduction: Plasmoblastic lymphoma (PBL) is a rare entity of non-Hodking lymphoma that usually occurs in the oral cavity in immunosuppressed patients. The involvement of other organs has rarely been reported. The breast can be the site of the initial manifestation of many diseases and in cases like this report, lymphomas should be considered as differential diagnoses. Case report: D.S., female, 28 years old, quality reviewer, married, two children. Carrier of human immunodeficiency virus (HIV) with recent treatment. Intern for investigation of liver, duodenal and breast abnormalities. She had nodules in both breasts, with progressive growth for about two months, in addition she presente nausea, vomiting, episodes of diarrhea and weight loss. On physical examination of the breasts, presence of apparent vascularization and asymmetric breast - right breast about two times the size of the contralateral. On palpation, the right breast presented with multiple hard and mobile nodules in a single left nodulation in an inferolateral quadrant measuring about $4 \mathrm{~cm}$, with the same characteristics as the previous ones. No axillary lymph node enlargement, supraclavicular or infraclavicular fossae were noticed. Core biopsy was performed on both breasts with the result of undifferentiated malignancy, with a diffuse standard and numerous foci of apoptosis (compatible with high-grade non-Hodgkin's lymphoma). In the immunohistochemical study, there was positivity for LCA, MUM 1, CD10, EMA, CMYC, CD30, CD3 and Ki-67 with a proliferative índex of 95\% in neoplasia. Histopathological aspects and immunohistochemical profile were compatible with plasmoblastic lymphoma. During hospitalization, the patient underwent a myelogram that showed slightly hypercellular bone marrow, hematopoiesis was present and was megaloblastic. There was moderate diffuse and a focal irregular infiltration by pleomorphic and dysmorphic plasma cells, suggesting spinal cord involvement by plasmoblastic lymphoma. In addition, oncological PET/CT was performed which showed a metabolic increase in nodular formations in the right breast and thickening of gastroduodenal transition compatible with the clinical information of lymphoproliferative disease. The patient underwent six cycles of the EPOCH polychemotherapy scheme (etoposide, prednisone, oncovinm, cyclophosphamide and ydroxideunorubicin), associated with four cycles of methotrexate at high doses, with remission of breast lesions and is currently being followed up. 